\title{
Comparison of the Antibacterial Activity of Australian Terminalia Spp. Extracts Against Klebsiella Pneumoniae: Novel Treatment for Ankylosing Spondylitis
}

lan Cock ( I.Cock@griffith.edu.au )

Griffith University https://orcid.org/0000-0002-8732-8513

Reece Courtney

Griffith University School of Science: Griffith University School of Natural Sciences

\section{Research Article}

Keywords: Australian plants, Combretaceae, autoimmune inflammatory disease, complementary and alternative therapies, Klebsiella pneumoniae, Kakadu plum, gubinge

Posted Date: November 22nd, 2021

DOI: https://doi.org/10.21203/rs.3.rs-1090934/v1

License: (c) (i) This work is licensed under a Creative Commons Attribution 4.0 International License. Read Full License 


\section{Abstract}

Traditional medicines prepared using Terminalia species have been used globally to treat inflammation and pathogenic infections. Recent studies have demonstrated that multiple Asian and African Terminalia spp. inhibit bacterial triggers of some autoimmune inflammatory disease, including ankylosing spondylitis. Despite this, the effects of Australian Terminalia spp. on a bacterial trigger of ankylosing spondylitis (K. pneumoniae) remain unexplored. Fifty-five extracts from five Australian Terminalia spp. were investigated for $K$. pneumoniae growth inhibitory activity. Methanolic, aqueous and ethyl acetate extracts of most species and plant parts inhibited $K$. pneumoniae growth, with varying potencies. Methanolic leaf extracts were generally the most potent bacterial growth inhibitors, with MIC values of $66 \mu \mathrm{g} / \mathrm{mL}$ (T. ferdinandiana), $128 \mu \mathrm{g} / \mathrm{mL}$ (T. carpenteriae) and $83 \mu \mathrm{g} / \mathrm{mL}$ (T. petiolares). However, the aqueous leaf extract was the most potent $T$. grandiflora extract (MIC $=87 \mu \mathrm{g} / \mathrm{mL}$ ). All $T$. catappa extracts displayed low growth inhibitory activity. The Terminalia spp. methanolic leaf extracts were examined by LC-MS and GC-MS. All contained a relative abundance of simple gallotannins (particularly gallic and chebulic acids), the flavonoid luteolin, as well as the monoterpenoids cineole and terpineol. Notably, all Terminalia spp. were non-toxic or of low toxicity in ALA and HDF toxicity assays, highlighting their potential for preventing the onset of ankylosing spondylitis and treating its symptoms once the disease is established.

\section{Introduction}

Ankylosing spondylitis (AS) is a relatively common autoimmune inflammatory disease that afflict approximately $0.9 \%$ of the world's population, with a significantly higher incidence in males (Rashid and Ebringer 2007). The onset is most common in middle to older age, although it can also occur in younger individuals (Braun and Sieper 2007; Rashid and Ebringer 2007). AS is a debilitating autoimmune inflammatory disease that affects spinal and pelvic sacroiliac joints of genetically susceptible individuals and is characterized by inflammation of the spinal joints, resulting in chronic back pain and reduced mobility. Chronic AS inflammation may induce ossification of the annulus fibrosis, resulting in the formation of syndesmophytes, which may form bone bridges between the vertebrae, thereby fusing them together. There currently are no effective cures for AS and current treatments instead target the symptoms of the disease (particularly pain and local tissue swelling) using analgesic and anti-inflammatory therapies rather than addressing the etiology or progression of the disease. However, these treatments only address the immediate symptoms of the disease and do not significantly affect progression of the disease or the significant tissue damage that results from chronic AS (Saag et al. 1994). Furthermore, prolonged usage of anti-inflammatory drugs and analgesics often results in toxicity and unwanted side effects (Saag et al. 1994).

Safe and effective AS chemotherapeutics are urgently required. Ideally, new AS drugs should prevent AS disease progression (rather than just blocking the symptoms), thereby inhibiting the formation of spinal syndesmophytes and subsequent vertebrae fusion. Notably, some causes of AS have been identified. It may be triggered by molecular mimicry in genetically susceptible individuals (individuals with the MHC class 1 allele HLA-B27) who contract $K$. pneumoniae infections (Cock and Cheesman 2018; Rashid and Ebringer 2007). Klebsiella pneumoniae infections induce the production of antibodies that target bacterial surface proteins, which also cross-react with host HLA-B27 QTDRED and DRED epitopes, as well collagens I, III and IV in genetically susceptible people (Rashid and Ebringer 2007). Binding of these bacterial-targeted antibodies to antigens in the host's tissues triggers inflammatory cascades, resulting in increased production of acute phase proteins, complement proteins, vasoactive amines, as well as multiple cytokines and chemokines (Rashid and Ebringer 2007). Furthermore, binding of the host-reactive antibodies to self-tissue also activates cellular processes, including activating natural killer cells, thereby stimulating further damage to the host tissue (Braun and Sieper 2007).

Multiple antibiotics are already effective inhibitors of $K$. pneumoniae growth, including the aminoglycosides, chloramphenicol, fluoroquinolones and tetracyclines. However, as AS is a chronic condition, preventative therapy would entail ongoing administration of antibiotics. This mode of therapy would be counter-productive as prolonged exposure to antibiotics induces the production of antibiotic resistant bacterial strains, thereby reducing (or even totally negating) the efficacy of the therapy. Indeed, several super-resistant bacterial strains of $K$. pneumoniae have already been reported, including a strain that is resistant to all common clinical antibiotics (Cheesman et al 2017; Ashayeri-Panah et al 2014). New safe and effective anti-K. pneumoniae therapies are urgently required. A re-examination of traditional medicines is an attractive option for antibiotic drug discovery as the anti-bacterial properties of some medicinal plants have been known for hundreds or (in some cases) thousands of years. Furthermore, due to the established use of traditional medicines, toxicities and side effects are often well documented. Plant-based preparations also often contain multiple compounds with antibacterial activity, as well as potentiating compounds, and are therefore essentially combinational therapies. This is noteworthy because the presence of multiple molecules targeting different aspects of bacterial growth substantially reduces the possibility of the bacteria developing resistance to the therapy. Indeed, we are unaware of any bacteria that have previously developed resistance to crude plant extracts.

Recent studies from our group have identified extracts prepared from several Terminalia spp. as being effective against the bacterial triggers of rheumatoid arthritis (RA) (Sirdaarta et al, 2015a; Cock and Van Vuuren 2014). Additionally, several other studies have also demonstrated that African and Indian Terminalia spp. are also good inhibitors of K. pneumoniae and are therefore promising leads for novel AS prevention and treatment therapies (Cock and Van Vuuren 2015; Cock 2015). In contrast, Australian Terminalia spp. are yet to be screened for growth inhibitory activity against bacterial triggers of AS, despite their taxonomic relationship with African and Indian species with noteworthy anti-K. pneumoniae activity and their inhibitory effects against bacterial triggers of RA (Cock 2015; Cock and Van Vuuren 2014), multiple sclerosis (MS) (Sirdaarta et al 2015b) and rheumatic fever (RF) (Wright et al 2016a). This study aimed to address this gap in the literature by screening several Australian Terminalia spp. for their inhibitory activity against $K$. pneumoniae using in vitro assays.

\section{Materials And Methods Plant sources and extraction}

All Terminalia ferdinandiana Exell., T. carpentariae C.T.White and T. grandiflora Benth. plant material was provided and identified by David Boehme of Northern Territory Wild Harvest, Australia and were sourced from wild trees in Kakadu Park, Australia under government collection licence. The Terminalia catappa L. 
bark, fruit and leaves were obtained from the Mt Cootha Botanical Gardens in Brisbane and were identified by the gardens botanist. Terminalia petiolaris Benth. fruit and leaves were a gift from Jacinta Monck of Kimberley Wild Gubinge, Australia. Voucher specimens of all plant materials were prepared and are stored at the School of Environment and Science, Griffith University, Australia. All plant materials were dried using a Sunbeam food dehydrator and the desiccated material was subsequently ground into a fine powder. Methanol, deionised water, ethyl acetate, chloroform or hexane were added individually to $1 \mathrm{~g}$ masses to give a final volume of $50 \mathrm{~mL}$ and extracted for $24 \mathrm{~h}$ at $4{ }^{\circ} \mathrm{C}$ with gentle mixing on an orbital shaker. All solvents were AR grade and were purchased from Ajax Fine-Chemicals, Australia. The mixtures were filtered through Whatman No. 54 filter paper to remove the solid plant material. The organic solvents were dried at $50{ }^{\circ} \mathrm{C}$ in a vacuum oven, whilst the aqueous extracts were lyophilised by at $-50^{\circ} \mathrm{C}$. The resultant dry pellets were weighed to determine extraction yield and were subsequently dissolved in $10 \mathrm{~mL}$ deionised water (containing $0.5 \%$ DMSO), filtered through a $0.22 \mu \mathrm{m}$ syringe driven filter (Sarstedt) and stored at $4^{\circ} \mathrm{C}$ until use.

\section{Qualitative phytochemical studies}

Alkaloids, cardiac glycosides, flavonoids, phenolic compounds, phytosterols, saponins, tannins and triterpenoids were detected using standard qualitative phytochemical assays (Cheesman et al 2019).

\section{Antibacterial screening}

\section{Test bacterial strains}

All media was supplied by Oxoid Ltd., Australia. The reference Klebsiella pneumoniae strain (ATCC31488) was purchased from American Type Culture Collection (ATCC). The clinical isolate Klebsiella pneumoniae strain was obtained from the science teaching laboratory at Griffith University, Australia. Stock cultures were subcultured and maintained in Mueller-Hinton broth at $4^{\circ} \mathrm{C}$. Culture purity was intermittently monitored by $16 \mathrm{~S}$ rRNA sequencing to ensure culture purity and homogeneity.

\section{Evaluation of antimicrobial activity}

The growth inhibitory properties of the Terminalia spp. extracts against $K$. pneumoniae were determined using standard disc diffusion assays (Cheesman et al 2019; Sirdaarta et al 2015a) on Mueller-Hinton agar plates. Each antimicrobial assay was performed three times, each with internal triplicates and mean values $( \pm$ SEM) are reported in this study. Standard discs of ampicillin $(10 \mu \mathrm{g})$ and chloramphenicol $(10 \mu \mathrm{g})$ were obtained from Oxoid Ltd., Australia and included on each agar plate as positive controls. Filter paper discs infused with $10 \mu \mathrm{L}$ of sterile deionised water were also included as a negative control.

\section{Minimum inhibitory concentration (MIC) determination}

The minimum inhibitory concentration for each extract was determined using liquid dilution MIC assay and solid phase (agar) MIC assays. Liquid dilution MIC assays are a common method for quantifying antibacterial activity and are considered sensitive compared to other assay systems (llanko and Cock 2019 ; Hübsch et al 2014). Furthermore, antibacterial evaluations using this method allows for comparison of the efficacy with other treatments and between studies. Solid phase agar disc MIC assays were also used in this study as a confirmatory assay, and as a more realistic approximation of bacterial infections on solid surfaces.

\section{Microplate liquid dilution MIC assay}

The MICs of the extracts were evaluated by previously described standard methods (llanko and Cock 2019; Hübsch et al, 2014). A $0.2 \mathrm{mg} / \mathrm{mL}$ volume of piodonitrotetrazolium violet (INT; Sigma, Australia) solution was used to indicate bacterial growth as a colour change (from clear to red). The MIC was visually determined as the lowest dose at which colour development was inhibited.

\section{Disc diffusion MIC assay}

The MICs of all extracts was also evaluated by disc diffusion assay across a range of extract concentrations as previously described (Cock et al 2015; Winnett et al 2014). Graphs of the zone of inhibition versus Ln concentration were plotted and linear regression was used to determine MIC values.

\section{Toxicity screening}

The toxicity of all extracts was assessed using the Artemia nauplii lethality assay (ALA) for rapid toxicity screening, as well as an MTS cellular proliferation assay for an evaluation of cytotoxicity.

\section{Artemia franciscana Kellogg nauplii toxicity screening}

Standard Artemia franciscana nauplii lethality assays were used as an initial evaluation of the toxicity of the Terminalia spp. extracts (Ruebhart et al 2009).

Potassium dichromate ( $1 \mathrm{mg} / \mathrm{mL}$ ) (AR grade, Chem-Supply, Australia) was included with each assay as a reference toxin. The $\mathrm{LC}_{50}$ with $95 \%$ confidence limits for each treatment was calculated using Probit analysis.

\section{Cellular viability assay}

All Terminalia spp. extracts were also screened for cytotoxicity against normal human primary dermal fibroblasts (HDF ATCC PCS-201-012) purchased from American Type Culture Collection. All extracts were screened at $200 \mu \mathrm{g} / \mathrm{mL}$ following $24 \mathrm{~h}$ exposure using standard protocols and absorbances were monitored at $540 \mathrm{~nm}$ and a blank wavelength of $690 \mathrm{~nm}$ using a Molecular Devices, Spectra Max M3 plate reader (Shalom and Cock, 2018). All tests were performed three times in triplicate $(n=9)$ and triplicate controls were included on each plate and the \% cellular viability (compared to the untreated control) was 
recorded. Cellular viability $\leq 50 \%$ of the untreated control was classified as toxic, whereas extracts that yielded cell viability $>50 \%$ untreated control were deemed to be nontoxic.

\section{HPLC-MS QTOF fingerprint analysis}

Separation, identification of extract components and determination of the relative abundance was achieved using an Agilent $1290 \mathrm{HPLC}$ system equipped with a Zorbax Eclipse C18 column ( $2.1 \times 100 \mathrm{~mm}, 1.8 \mu \mathrm{m}$ particle size) linked to an Agilent 6530 quadrupole time-of-flight (QTOF) mass spectrometer. The chromatographic separation utilised parameters previously optimised for the identification of phytochemical components in related Terminalia spp. extracts (Sirdaarta et al 2015a). Mass spectra were obtained in the electron ionisation mode and analytes were recorded for 45 mins in total ion count (TIC) mode.

\section{GC-MS head space analysis}

The Terminalia spp. extract components were separated, identified and their relative abundance was analysed using a Shimadzu GC-2010 plus (USA) chromatography system linked to a Shimadzu MS TQ8040 (USA) mass selective detector system using previously developed parameters (Sirdaarta et al $2015 \mathrm{~b}$ ). For analysis, the mass spectrometer's ionisation mode was set to $70 \mathrm{eV}$ and the mass signals were recorded as total ion count (TIC) mode for 45 mins across a mass range of $45-450 \mathrm{~m} / \mathrm{z}$.

\section{Statistical analysis}

Data is expressed as the mean \pm SEM of three independent experiments, each with three internal replicates $(n=9)$. Statistical significance was calculated using one-way analysis of variance (ANOVA) followed by Tukey's post hoc analysis. $P$ values $<0.01$ were considered significant.

\section{Results}

\section{Liquid extraction yields}

Extraction of the Terminalia spp. plant materials with solvents of varying polarity yielded dried plant extracts ranging from $32 \mathrm{mg}$ (T. grandifloria leaf ethyl acetate extract) to $426 \mathrm{mg}$ (aqueous $T$. ferdinandiana leaf extract) from $1 \mathrm{~g}$ of dried, powdered plant material. These dried extracts were resuspended in $10 \mathrm{~mL}$ deionised water (containing $1 \%$ DMSO) for further testing. The aqueous and methanolic extracts generally had substantially higher yields of extracted material compared to the corresponding chloroform, ethyl acetate and hexane extracts (Table 1). Additionally, the higher polarity methanol and water solvent extractions yielded the greatest relative abundance and widest diversity of phytochemical classes for all Terminalia spp. across all plant materials examined. In general, all of the methanolic and aqueous Terminalia spp. extracts contained high levels of phenolics, flavonoids and tannins, as well as moderate levels of saponins. The ethyl acetate extracts also contained similar phytochemical classes, although in substantially lower relative abundances compared to the methanolic and aqueous extracts.

\section{Antibacterial activity}

To evaluate the growth inhibitory activity of the Australian Terminalia spp. extracts against $K$. pneumoniae, all extracts were initially screened by disc diffusion assays and the zones of inhibition (ZOI) were measured. The methanolic, aqueous and ethyl acetate T. ferdinandiania fruit (Figure 1a), leaf (Figure 1b) and seed (Figure 1c) extracts all inhibited K. pneumoniae growth. Notably, the T. ferdinandiania leaf extracts were substantially stronger than the extracts produced from either the fruit or seeds of that species (as evaluated by size of the ZOI). Indeed, ZOIs of approximately 18 mm were measured for the methanolic leaf extract against both the reference and clinical isolates strains of $K$. pneumonia (Figure $1 \mathrm{~b}$ ). Furthermore, this extract was a substantially better inhibitor of $K$. pneumoniae growth than both control antibiotics. Both strains of $K$. pneumonia were completely resistant to the ampicillin control, although both bacterial strains were highly susceptible to chloramphenicol (ZOls $\sim 14 \mathrm{~mm}$ ). Interestingly, the ZOI measured for the methanolic T. ferdinandiania leaf extract was substantially larger than the chloramphenicol control disc, indicating that the extract is a substantially more potent anti-K. pneumoniae treatment than the pure antibiotic. Additionally, a relatively high dose $(10 \mu \mathrm{g})$ of pure chloramphenicol was infused into the control antibiotic disc. In contrast, the extracts tested herein contain complex mixtures of compounds, with the antibacterial components expected to account for a relatively low percentage of the total extracted material. Therefore, the inhibitory activity of the individual methanolic T. ferdinandiania leaf extract components are particularly noteworthy. In contrast, the methanolic $T$. ferdinandiana fruit and seed extracts produced ZOls of approximately 10 and $7 \mathrm{~mm}$ respectively.

The methanolic T. carpenteriae leaf extract was also a strong inhibitor of both K. pneumoniae strains (ZOls 12 mm) (Figure 2). Indeed, the K. pneumoniae growth inhibition by this extract compares favourably to the inhibition by the antibiotic controls (as judged by the ZOI size). Similarly, the methanolic $T$. grandiflora fruit (Figure 3a) and leaf (Figure 3b) extracts were also good inhibitors of the growth of both K. pneumoniae strains, with ZOls of approximately 11 and $13 \mathrm{~mm}$ respectively. However, a notable difference were evident for the $T$. grandiflora leaf extracts compared to fruit extract (and to the other Terminalia spp. screened in our study). Specifically, the aqueous T. grandiflora leaf extract was a substantially stronger inhibitor of both $K$. pneumoniae strains than the methanolic extract was, with ZOls of 16 and $16.7 \mathrm{~mm}$ against the reference and clinical isolate strains respectively.

The methanolic T. petiolares fruit (Figure $4 \mathrm{a}$ ) and leaf extracts (Figure 4b) were also strong inhibitors of both K. pneumoniae strains (ZOls of approximately 13.5 and $17 \mathrm{~mm}$ respectively). The aqueous and ethyl acetate $T$. petiolares fruit and leaf extracts were also good inhibitors of bacterial growth, albeit with substantially smaller ZOIs than measured for the corresponding methanolic extracts. In contrast, relatively weak anti- $K$. pneumoniae activity was noted for the T. catappa bark (Figure 5a), fruit (Figure $5 \mathrm{~b}$ ) and leaf extracts (Figure $5 \mathrm{c}$ ). Indeed, weak growth inhibition was only seen for the methanolic T. catappa extracts (ZOls $\leq 7.5 \mathrm{~mm}$ against both $K$. pneumoniae strains), whilst all other $T$. catappa extracts were completely ineffective inhibitors of $K$. pneumoniae growth.

\section{Quantification of minimum inhibitory concentration (MIC)}


The relative level of $K$. pneumoniae growth inhibitory activity was also quantified and further evaluated by determination of MIC values of each extract (Table 2). MIC values were only determined for those extracts that displayed activity in the disc diffusion screening assays. With some noteworthy exceptions, the methanolic and ethyl acetate leaf extracts were generally substantially more potent inhibitors of $K$. pneumoniae growth than extracts produced from other plant parts, or using other solvents. The $T$. ferdinandiana methanolic (66 and $53 \mu \mathrm{g} / \mathrm{mL}$ against the reference and clinical strains respectively) and ethyl acetate leaf extracts ( 78 and $75 \mu \mathrm{g} / \mathrm{mL}$ against the reference and clinical strains respectively) were particularly good inhibitors of both $K$. pnuemoniae strains. Similarly, the T. petiolares leaf methanolic ( 83 and $69 \mu \mathrm{g} / \mathrm{mL}$ against the reference and clinical strains respectively) and ethyl acetate extracts (105 and 92 $\mu \mathrm{g} / \mathrm{mL}$ against the reference and clinical strains respectively) were also noteworthy. As $K$. pneumoniae can trigger ankylosing spondylitis in genetically susceptible people (Cock and Cheesman 2018), these extracts may be particularly promising for the prevention and control of that disease, as well as other diseases caused by $K$. pneumoniae infections.

The growth inhibitory activity of the methanolic and ethyl acetate extracts of most parts of most of the other Terminalia spp. was also noteworthy, with MIC values generally substantially below $1000 \mu \mathrm{g} / \mathrm{mL}$. The $T$. catappa extracts (prepared using all plant parts) were a notable exception to this trend, with high MIC values $(1500->5000 \mu \mathrm{g} / \mathrm{mL})$ determined for all $T$. catappa extracts against both $K$. pnuemoniae strains. Similarly, MIC values substantially $>1000 \mu \mathrm{g} / \mathrm{mL}$ were also determined for all of the T. ferdinandiana seed extracts against these bacteria. Thus, the T. catappa extracts and the $T$. ferdinandiana seed extracts are likely to be of limited use for the prevention and treatment of ankylosing spondylitis. A further trend was also evident: The leaf extracts were generally substantially more potent inhibitors of $K$. pneumoniae growth than the corresponding fruit, bark or seed extracts. Additionally, the anti-K. pneumoniae activity of the aqueous extracts of most Terminalia spp. and plant parts studied were also noteworthy, albeit with substantially higher MIC values than determined for the methanolic and ethyl acetate extracts.

\section{Quantification of toxicity}

All of the Australian Terminalia spp. extracts were initially screened across a range of concentrations in the Artemia nauplii toxicity assay and $\mathrm{LC}_{50}$ values were quantified (Table 2). As $L_{5}$ values $>1000 \mu \mathrm{g} / \mathrm{mL}$ have been defined as nontoxic in this assay (Ruebhart et al 2009), the majority of the Australian Terminalia spp. extracts were classified as nontoxic. Indeed, only the methanolic T. ferdinandiana fruit $\left(\mathrm{LC}_{50}=925 \mu \mathrm{g} / \mathrm{mL}\right), T$. grandiflora fruit $\left(\mathrm{LC}_{50}=934\right.$ $\mu \mathrm{g} / \mathrm{mL})$ and leaf $\left(\mathrm{LC}_{50}=884 \mu \mathrm{g} / \mathrm{mL}\right)$, as well as the $T$. petiolares fruit extracts $\left(\mathrm{LC}_{50}=925 \mu \mathrm{g} / \mathrm{mL}\right)$ displayed toxicity. Furthermore, the relatively high $\mathrm{LC}_{50}$ values calculated for those extracts indicate that they are only of low toxicity. Additionally, it is noteworthy that whilst Artemia nauplii are generally robust, they are sensitive to acidic pH (Kwast, 1996) and extracts containing high levels of ascorbic acid may provide falacious results (Wright et al., 2016b; Kwast, 1996). Notably, high ascorbic acid content is a characteristic observed in all Terminalia spp. (Cock, 2015) and previous studies have reported that the apparent toxicity of Indian (Mandeville and Cock, 2018), Australian (Shalom and Cock, 2018; Lee et al., 2016; Wright et al., 2016b; Sirdaarta et al., 2015a, b) and African Terminalia spp. (Cock and van Vuuren 2015; Cock and van Vuuren 2014) correlates with their high ascorbic acid content. Thus, this assay may have overestimated the toxicity of these extracts.

Cellular cytotoxicity assays are generally considered to provide a more relevant evaluation of the toxicity of plant extracts. Notably, acidic phytochemicals (including ascorbic acid) generally do not significantly influence the viability of eukaryotic cell lines and thus we also evaluated the toxicity of the Australian Terminalia spp. extracts using normal human dermal fibroblasts HDF viability assays. Notably, exposure of HDFs to all of the Terminalia spp. extracts (including those classified as low toxicity in the Atremia nauplii assay) resulted in substantially $>50 \%$ for all tests (compared to untreated control viability). Thus, all extracts were determined to be nontoxic to HDFs.

\section{Phytochemical fingerprint analysis}

The T. ferdinandiana, T. carpentariae, T. grandiflora and T. petiolares methanolic leaf extracts generally displayed substantially greater bacterial inhibitory activity than the other solvent extractions (or the T. catappa extracts). Thus, those extracts were analysed by metabolomic phytochemical fingerprint analysis. In recent studies, LC-MS analyses have been used to profile the phytochemical composition of multiple Terminalia spp. extracts for the presence of specific compounds, and to quantify their relative abundances in those extracts (Nel et al 2020; Rayan et al 2015; Sirdaarta et al 2015a; Sirdaarta et al 2015b). Notably, a number of compounds were detected in high relative abundance in the leaf extracts of multiple Terminalia spp. leaf extracts. In particular, the low molecular mass tannins ellagic acid and ellagic acid dehydrates, gallic acid, chebulic acid, and exifone were present in relative abundance. Additionally, the higher molecular mass tannins castalagin, chebulagic acid, chebulinic acid and corilagin were also previously reported in relative abundance in the leaf extracts of other Terminalia spp. leaf extracts. The flavonoid luteolin was also previously reported to be in relative abundance in multiple Terminalia spp. leaf extracts (Nel et al 2020; Rayan et al 2015; Sirdaarta et al 2015a; Sirdaarta et al 2015b).

Notably, all of these compounds were also identified in relative abundance in the Australian Terminalia spp. leaf extracts examined in our study (Table 3). Gallotannins were particularly prevalent across all of the Australian Terminalia spp. methanolic leaf extracts, with the levels of gallic acid (5.8-6.9\% relative abundance), chebulic acid (4-6.9\% relative abundance), corilagin (5-6.9\% relative abundance), chebulinic acid (3.1-4.5\% relative abundance) and exifone (5.7$6.6 \%$ relative abundance) particularly noteworthy. The percentages cited in Table 3 correspond to the chromatographic percent peak area compared to the total area for all peaks in the chromatogram. The relative abundances of ellagitannins was also notable, albeit generally at lower levels than noted for the gallotannins. In particular, ellagic acid (1.3-1.7\% relative abundance), ellagic acid dehydrates (2.1-2.8 \% relative abundance) and castilagin (2.1-2.8 \% relative abundance) were present across the extracts of all of the Australian Terminalia spp. studied herein. The flavonoid luteolin was also present in particularly high relative abundance (5.8-7.4\%) across all of the extracts tested. Notably, the relative abundances of the tannin and flavonoid compounds in the methanolic $T$. ferdinandiana, T. carpentariae and T. grandiflora leaf extracts in our study correlate closely with the previously reported values (Cock et al 2019). To the best of our knowledge, the presence and abundance of these compounds has not previously been reported in T. petiolares. Interestingly, the LC-MS fingerprint of the $T$. petiolares leaf extract reported herein is similar in both identity and relative percentage abundance to the other Australian Terminalia spp. leaf extracts. 
Previous studies have also identified a relative abundance of monoterpenoids in Terminalia spp. leaf extracts using GC-MS headspace techniques (Wright et al., 2016b; Sirdaarta et al., 2015b). In particular, cineole, terpineol, borneol, limonene have all been reported in relative abundance, with camphor, isomenthol, and isomyocorene reported in lower relative abundance. Therefore, our study also screened for these compounds using GC-MS headspace fingerprint analysis to identify and quantify the relative abundance these compounds in the T. ferdinandiana, T. carpentariae, T. grandiflora and T. petiolares methanolic leaf extracts. Interestingly, cineole (1.6-2.2 \% relative abundance), terpineol (1.3-1.6 \% relative abundance), borneol (0.9-1.5\% relative abundance), limonene ( 0.4 $\%$ relative abundance) and camphor (0.3-0.5\% relative abundance) were identified in particularly high relative abundance in all of the extracts screened herein. Notably, these relative abundances are also similar to the levels previously reported for other Terminalia spp. leaf extracts using similar analytical methods (Wright et al 2019).

\section{Discussion}

The current repertoire of therapies to treat AS are not ideal and only target the symptoms of the disease, whilst allowing irreversible tissue degradation to continue in the vertebral and sacroiliac joints. Therapies targeting the etiological events would prevent the onset of the disease, which would not only alleviate the symptoms of AS, but would also prevent tissue damage. The role of $K$. pneumoniae infections in the etiology of AS is well established and is supported by a wealth of evidence (Cock and Cheesman 2018; Rashid and Ebringer 2007). Despite this, there are currently no clinical therapeutics for AS that target $K$. pneumoniae. Inhibiting the growth of $K$. pneumoniae using prophylactic antibiotic therapies may be effective in the prevention and treatment of AS, and other autoimmune inflammatory diseases. However, prophylactic usage of conventional antibiotics would also induce the development of further antibiotic resistance, rendering the target bacterium (and other microbial species) unsusceptible to the effects of the antibiotic. The use of combinations of antibiotics (or antibiotics and potentiator compounds) may be more effective than antibiotic monotherapies and may also decrease the possibility of the bacteria developing further resistance.

Traditional medicines and herbal therapies are promising targets for antibiotic drug discovery as they have often been used for long periods (in some cases, thousands of years) and are perceived (often erroneously) to be safer than synthetic drugs (Moreira et al 2014). The development of multiple-drug resistant strains of many clinically relevant bacterial pathogens has resulted in the failure of many current antibiotics and has highlighted the need to develop new safe and effective antibiotic chemotherapeutics. Indeed, the World Health Organisation (WHO) considers the development of super-resistant bacteria to be one of the biggest challenges currently facing medical science (WHO, 2014). Previously, new antibiotic therapies have readily been obtained from microbial sources, and from chemical modification of natural product scaffolds. However, for reasons discussed elsewhere (Cheesman et al 2017), it is unlikely that these methods of drug development will be as fruitful in the future and new development methods are needed.

The genus Terminalia is an attractive target for the development of novel antibacterial therapies as multiple Terminalia spp. have been used traditionally to treat both inflammation and bacterial infections (Cock 2015). Furthermore, extracts produced from several Terminalia spp. have been reported to have noteworthy activity against a wide range of bacterial pathogens (Nel et al 2020; Mandeville and Cock 2018; Courtney et al 2015), including the K. pneumoniae trigger of AS (Mandeville and Cock 2018; Cock and Van Vuuren 2015). Whilst the bacterial growth inhibitory properties of several Australian Terminalia spp. have been reported in recent years (Wright et al 2019; Sirdaarta et al 2015a; Sirdaarta et al 2015b), they have yet to be tested for anti-K. pneumoniae activity. Methanolic extracts prepared from all of the Terminalia spp. screened in our study displayed noteworthy $K$. pneumoniae growth inhibitory activity. Additionally, the aqueous and ethyl acetate extracts of all Terminalia spp. tested (except T. catappa) also inhibited K. pneumoniae growth. Notably, for all of the Australian Terminalia spp., the leaf extracts had substantially greater potency than the corresponding fruit, bark or seed extracts. Therefore, the methanolic $T$.

ferdinandiana, T. carpentariae, T. grandiflora and T. petiolares leaf extracts were further analysed to determine their phytochemical composition using both LCMS and GC-MS headspace analysis.

Metabolomic fingerprint analyses of the methanolic T. ferdinandiana, T. carpentariae, T. grandiflora and T. petiolares leaf extracts confirmed the presence of some noteworthy compounds with therapeutically relevant properties. In particular, our study targeted the tannins, flavonoids and volatile terpenoids. All of the methanolic leaf extracts evaluated contained a diversity of tannins in relative abundance. Notably, tannins inhibit the growth of multiple bacterial pathogen growth by disrupting bacterial cell walls (Hogg and Embery 1982; Wu-Yuan et al 1988), binding and inactivating specific bacterial cell surface proteins, as well as functioning as inhibitors of bacterial enzymes (Wu-Yuan et al 1988; Buzzini et al 2008). Thus, it is likely that the Australian Terminalia spp. tannins components detected in our study may contribute to the anti-K. pneumoniae activity reported herein. Additionally, the flavonoid luteolin was also identified in all of the Australian Terminalia spp. methanolic leaf extracts examined in our study. Interestingly, multiple flavonoids including luteolin have been reported to have potent broad-spectrum antibacterial activity (Cushnie and Lamb 2005; Resende et al 2018) and may therefore also contribute to the noteworthy antibacterial activity reported herein.

Another feature of the Australian Terminalia spp. phytochemistry reported herein was the diversity and relative abundance of volatile terpenoids identified in the methanolic leaf extracts. In particular, the monoterpenoids cineole, terpineol and linalool were present in relative abundance. Interestingly, each of these compounds has potent broad-spectrum bacterial growth inhibitory activity against both gram positive and gram negative pathogens (Zenegin and Baysal, 2014; Paduch et al, 2007). Their antibacterial activities are linked to their small sizes and lipophilic characteristics (Paduch et al, 2007). Cineole, terpineol and linalool (as well as other monoterpenoids) can insert into cytosolic membranes, thereby altering bacterial membrane fluidity and permeability, as well as changing the conformation and function of bacterial membrane proteins. These membrane changes dramatically alter bacterial respiration, as well as several other crucial cellular processes (Cristani et al 2007; Di Pasqua et al 2006). Furthermore, modulation of bacterial membrane fluidity by small volatile terpenoids may also alter the permeability of the membrane to other compounds with growth inhibitory properties, thereby potentiating their antibiotic effects (BremStecher and Johnson 2003).

The diversity and abundance of small volatile monoterpenoids in the Australian Terminalia spp. extracts is also particularly noteworthy as their non-specific antibacterial mechanisms are inherently difficult for bacteria to adapt to and to develop resistance against. Indeed, we were unable to find any reports of the 
development of resistance to monoterpenoids by any bacterial species. Furthermore, multiple terpenoid compounds that may function via several antibacterial mechanisms were identified in these extracts. These combinations may enhance the growth inhibitory potency of the combination relative to the individual components, and may also substantially reduce the development of antibiotic resistance in the target bacteria. It is unlikely any of these compounds is solely responsible for the bacterial growth inhibitory activity reported in our study. Instead, it is likely that multiple components with several antibacterial properties may collectively interact to produce the noteworthy activity reported herein. Furthermore, synergistic interactions may potentiate the anti- $K$. pneumoniae activity, thereby increasing their efficacy of the individual components.

Our study was limited to examining the inhibitory activity of the Australian Terminalia spp. extracts towards a bacterial trigger of AS. However, these are crude extracts, which contain complex phytochemical mixtures. It is therefore possible that these extracts may also modulate other inflammatory processes such as cytokine release and therefore effect several aspects of AS disease progression. Indeed, several of the compounds identified in our study have been reported to have pleuripotent anti-inflammatory effects by acting on both the initiator and downstream inflammatory stages of the disease. For example, several of the terpenoids that we have identified herein can suppress NF-KB signalling, and may therefore also directly inhibit inflammation (Salminen et al, 2008). Further studies are required to evaluate Australian Terminalia spp. extracts for direct anti-inflammatory properties, and for additional combinational effects that may enhance the therapeutic properties of individual components of these extracts.

\section{Conclusions}

The results of this study demonstrate the potential of T. ferdinandiana, T. carpentariae, T. grandiflora and T. petiolares as inhibitors of K. pneumoniae, a bacterial trigger of AS. The methanolic and ethyl acetate extracts prepared from T. ferdinandiana and T. petiolaris leaves were particularly good inhibitors of $K$. pneumoniae growth. Additionally T. ferdinandiana, T. grandiflora and T. petiolaris fruit extracts, as well several aqueous extracts, also had noteworthy activity. Whilst the extracts examined in this report are promising as anti-AS agents, caution is needed before these compounds can be applied to medicinal purposes. In particular, further toxicity studies using human cell lines are needed to verify the suitability of these extracts for these purposes.

\section{Declarations}

\section{ACKNOWLEDGEMENTS}

We are grateful to David Boehme of Northern Territory Wild Harvest, Australia for providing the T. ferdinandiana, T. carpentaria and T. grandiflora plant material used in these studies. We also thank Jacinta Monck of Kimberley Wild Gubinge, and the Mount Cootha Botanical Gardens for providing the T. petiolaris and T. catappa plant materials respectively. Financial support for this work was provided by the Centre for Planetary Health and Food Security, and the School of Environment and Science, Griffith University, Australia.

\section{References}

1. Ashayeri-Panah M, Feizabadi MM, Eftekhar F (2014) Correlation of multi-drug resistance, integron and blaESBL gene carriage with genetic fingerprints of extended-spectrum $\beta$-lactamase producing Klebsiella pneumoniae. Jundishapur J Microbiol 7(2):e8747

2. Braun J, Sieper J (2007) Ankylosing spondylitis. Lancet 369: 1379-1390

3. Brem-Stecher B, Johnson EA (2003) Sensitisation of Staphylococcus aureus and Escherichia coli to antibiotics by the sesquiterpenoids nerolidol, farnesol, bisabolol, and apritone. Antimicrob Agents Chemother 47(10):3357-3360

4. Buzzini P, Arapitsas P, Goretti M et al (2008) Antimicrobial and antiviral activity of hydrolysable tannins. Mini Rev Med Chem8: 1179-1187

5. Cheesman MJ, llanko A, Blonk B, et al (2017) Developing new antimicrobial therapies: Are synergistic combinations of plant extracts/compounds with conventional antibiotics the solution? Pharmacogn Rev 11: 57-72

6. Cheesman MJ, White A, Matthews B, et al (2019) Terminalia ferdinandiana fruit and leaf extracts inhibit methicillin-resistant Staphylococcus aureus growth. Planta Med 85(16): 1253-1262

7. Cock IE, Wright MH, Matthews B, et al (2019) Bioactive compounds sourced from Terminalia spp. in bacterial malodour prevention: an effective alternative to chemical additives. Int J Cosmet Sci 41(5): 496-508

8. Cock IE (2015) The medicinal properties and phytochemistry of plants of the genus Terminalia (Combretaceae). Inflammopharmacol 23(5): 203-229

9. Cock IE, Cheesman MJ (2018) The potential of plants of the genus Syzygium (Myrtaceae) for the prevention and treatment of arthritic and autoimmune diseases. In Bioactive Foods as Dietary Interventions for Arthritis, Osteoarthritis, and related Autoimmune Diseases, $2^{\text {nd }}$ Edition. Editors Preedy VR, Watson RR. Elsevier Publishing

10. Cock IE, Winnett V, Sirdaarta J, et al (2015) The potential of selected Australian medicinal plants with anti-Proteus activity for the treatment and prevention of rheumatoid arthritis. Pharmacogn Mag 11(42): S190-S208

11. Cock IE, van Vuuren SF (2015) The potential of selected some South African plants with anti-Klebsiella activity for the treatment and prevention of ankylosing spondylitis. Inflammopharmacol 23(1): 21-35

12. Cock IE, van Vuuren SF (2014) Anti-Proteus activity of some South African medicinal plants: Their potential for the treatment and prevention of rheumatoid arthritis. Inflammopharmacol 21(1): 23-36

13. Courtney R, Sirdaarta J, Matthews B, et al (2015) Tannin components and inhibitory activity of Kakadu plum leaf extracts against microbial triggers of autoimmune inflammatory diseases. Pharmacogn J 7(1): 18-31 
14. Cristani M, D’Arrigo M, Mandalari G, et al (2007) Interaction of four monoterpenes contained in essential oils with model membranes: implications for their antibacterial activity. J Agric Food Chem 55: 6300-6308

15. Cushnie TT, Lamb AJ (2005) Antimicrobial activity of flavonoids. Int J Antimicrob Agents 26: 343-356

16. Di Pasqua R, Hoskins N, Betts G, et al (2006) Changes in membrane fatty acid composition of microbial cells induced by addition of thymol, carvacrol, limonene, cinnamaldehyde, and eugenol in the growing media. J Agric Food Chem 54: 2745-2749

17. Hogg S, Embery G (1982) Blood-group-reactive glycoprotein from human saliva interacts with lipoteichoic acid on the surface of Streptococcus sanguis cells. Arch Oral Biol27: 261-268

18. Hübsck Z, Van Zyl RL, Cock IE, et al (2014) Interactive antimicrobial and toxicity profiles of conventional antimicrobials with Southern African medicinal plants. S Afr J Bot 93: 185-197

19. Ilanko A, Cock IE (2019) The interactive antimicrobial activity of conventional antibiotics and Petalostigma spp. extracts against bacterial triggers of some autoimmune inflammatory diseases. Pharmacogn J 11(2): 292-309

20. Kwast KE, Hand SC (1996) Oxygen and pH regulation of protein synthesis in mitochondria from Artemia franciscana embryos. Biochem J313: 207-213

21. Lee CJ, Wright MH, Arnold MSJ, et al (2016) Inhibition of Streptococcus pyogenes growth by native Australian plants: New approaches towards the management of impetigo, pharyngitis and rheumatic heart disease. Pharmacogn Commun 6(3): 164-173

22. Mandeville A, Cock IE (2018) Terminalia chebula Retz. fruit extracts inhibit bacterial triggers of some autoimmune diseases and potentiate the activity of tetracycline. Indian J Microbio. 58: 496-506

23. Moreira DD, Teixeira SS, Monteiro MH, et al (2014) Traditional use and safety of herbal medicines. Rev Bras Farmacogn 24: $248-257$

24. Nel AL, Murhekar, S, Matthews, B, et al (2020) The interactive antimicrobial activity of Terminalia sericea Burch ex DC. leaf extracts and conventional antibiotics against bacterial triggers of selected autoimmune inflammatory diseases. S Afr J Bot 133: 17-29.

25. Paduch R, Kandefer-Szerszen M, Trytek M et al (2007) Terpenes: substances useful in human healthcare. Arch Immunol Ther Exp 55: 315-327

26. Rashid T, Ebringer A (2007) Ankylosing spondylitis is linked to Klebsiella - the evidence. Clin Rheumatol 26: 858-864

27. Rayan P, Matthews B, McDonnell A, et al (2015) Terminalia ferdinandiana extracts as inhibitors of Giardia duodenalis proliferation: a new treatment for giardiasis. Parasitol Res 114: 2611-2620.

28. Resende FA, Nogueira LG, Bauab TM, et al (2018) Antibacterial potential of flavonoids with different hydroxylation patterns. Eclet Quim 40: $173-179$

29. Ruebhart DR, Wickramasinghe W, Cock IE (2009) Protective efficacy of the antioxidants vitamin E and Trolox against Microcystis aeruginosa and microcystin-LR in Artemia franciscana nauplii. J Toxicol Environ Health Part A 72: 1567-1575.

30. Saag KG, Koehnke R, Caldwell JR, et al (1994) Low dose long-term corticosteroid therapy in rheumatoid arthritis: an analysis of serious adverse events. Am J Med 96(2): 115-123

31. Salminen A, Lehtonen M, Suuronen Tet al (2008) Terpenoids: Natural inhibitors of NF-kB signalling with anti-inflammatory and anticancer potential. Cell Mol Life Sci 65(19): 2979-2999

32. Shalom J, Cock IE (2018) Terminalia ferdinandiana Exell. fruit and leaf extracts inhibit proliferation and induce apoptosis in selected human cancer cell lines. Nutr Cancer 70(4): 579-593.

33. Sirdaarta J, Matthews B, Cock IE (2015a) Inhibitory activity of Kakadu plum fruit extracts against microbial triggers of rheumatoid arthritis: Identification of stilbene and tannin components. J Funct Food 17: 610-620

34. Sirdaarda J, Matthews B, White A, et al (2015b) GC-MS and LC-MS analysis of Kakadu plum fruit extracts displaying inhibitory activity against microbial triggers of multiple sclerosis. Pharmacogn Commun 5(2): 100-115

35. WHO (2014) The Evolving Threat of Antimicrobial Resistance: Options for Action. World Health Organization 2014.

http://apps.who.int/iris/bitstream/10665/44812/1/9789241503181_eng.pdf. Accessed 14 March 2017.

36. Winnett V, Boyer H, Sirdaarta J, et al (2014) The potential of Tasmannia lanceolata as a natural preservative and medicinal agent: antimicrobial activity and toxicity. Pharmacogn Commun 4(1): 42-52

37. Wright MH, Shalom J, Matthews B, et al (2019) Terminalia ferdinandiana Exell: extracts inhibit Shewanella spp. growth and prevent fish spoilage. Food Microbiol 78: 114-122

38. Wright MH, Arnold MSJ, Lee CJ, et al (2016a) Qualitative phytochemical analysis and antibacterial activity evaluation of Indian Terminalia spp. against the pharyngitis causing pathogen Streptococcus pyogenes. Pharmacogn Commun 6(2): 85-92

39. Wright MH, Sirdaarta J, White A, et al (2016b) GC-MS headspace analysis of Terminalia ferdinandiana fruit and leaf extracts which inhibit Bacillus anthracis growth. Pharmacogn J9: 73-82

40. Wu-Yuan C, Chen C, Wu R (1988) Gallotannins inhibit growth, water-insoluble glucan synthesis, and aggregation of Mutans streptococci. J Dent Res67: $51-55$

41. Zenegin H, Baysal AH (2014) Antibacterial and antioxidant activity of essential oil terpenes against pathogenic and spoilage-forming bacteria and cell structure-activity relationships evaluated by SEM microscopy. Molecules 19: 17773-17798.

\section{Tables}

Due to technical limitations, table 1 is only available as a download in the Supplemental Files section. 
Table 2: Disc diffusion (DD) and liquid dilution (LD) MIC values $(\mu \mathrm{g} / \mathrm{mL})$ for Australian Terminalia spp. extracts against $K$. pneumoniae, and $L C_{50}(\mu \mathrm{g} / \mathrm{mL})$ in the ALA and HDF assays.

\begin{tabular}{|c|c|c|c|c|c|c|c|}
\hline \multirow[t]{2}{*}{ Plant Species } & \multirow[t]{2}{*}{ Extract } & \multicolumn{2}{|c|}{ K. pneumoniae (ATCC31488) } & \multicolumn{2}{|c|}{ K. pneumoniae (clinical strain) } & \multicolumn{2}{|l|}{$\mathrm{LC}_{50}$} \\
\hline & & DD MIC & LD MIC & DD MIC & LD MIC & ALA & HDF \\
\hline \multirow[t]{9}{*}{ T. ferdinandiana } & Fruit M & 653 & 416 & 825 & 508 & 925 & - \\
\hline & Fruit W & 584 & 345 & 620 & 482 & 1164 & - \\
\hline & Fruit E & 406 & 227 & 463 & 286 & 1083 & - \\
\hline & Leaf $M$ & 253 & 66 & 220 & 53 & 1172 & - \\
\hline & Leaf $W$ & 584 & 290 & 459 & 221 & 1428 & - \\
\hline & Leaf $\mathrm{E}$ & 285 & 78 & 224 & 75 & 1026 & - \\
\hline & Seed M & 1434 & 1346 & 1235 & 828 & 1863 & - \\
\hline & Seed W & 1146 & 922 & 1422 & 1139 & 2295 & - \\
\hline & Seed E & 1287 & 1046 & 1046 & 1047 & 1940 & - \\
\hline \multirow[t]{3}{*}{ T. carpenteriae } & Leaf M & 558 & 128 & 492 & 98 & 1185 & - \\
\hline & Leaf W & 1458 & 983 & 1288 & 725 & 1385 & - \\
\hline & Leaf $\mathrm{E}$ & 825 & 260 & 610 & 164 & 1553 & - \\
\hline \multirow[t]{6}{*}{ T. grandiflora } & Fruit M & 1235 & 983 & 1492 & 1146 & 934 & - \\
\hline & Fruit W & 1150 & 884 & 1280 & 1003 & 1387 & - \\
\hline & Fruit E & 1635 & 1220 & 1587 & 1322 & 1876 & - \\
\hline & Leaf M & 854 & 526 & 527 & 392 & 884 & - \\
\hline & Leaf W & 399 & 87 & 304 & 198 & 2564 & - \\
\hline & Leaf $\mathrm{E}$ & 883 & 600 & 488 & 274 & - & - \\
\hline \multirow[t]{6}{*}{ T. petiolaris } & Fruit M & 733 & 427 & 651 & 376 & 916 & - \\
\hline & Fruit W & 946 & 669 & 827 & 658 & 1257 & - \\
\hline & Fruit E & 870 & 364 & 693 & 274 & 1073 & - \\
\hline & Leaf $M$ & 304 & 83 & 228 & 69 & 1120 & - \\
\hline & Leaf W & 725 & 342 & 655 & 253 & 1362 & - \\
\hline & Leaf $\mathrm{E}$ & 382 & 105 & 327 & 92 & 1225 & - \\
\hline \multirow[t]{3}{*}{ T. catappa } & Bark M & 4385 & 3760 & 4140 & 3406 & 1473 & - \\
\hline & Fruit M & $>5000$ & $>5000$ & $>5000$ & $>5000$ & 1088 & - \\
\hline & Leaf $M$ & 2856 & 1685 & 2540 & 1455 & 1227 & - \\
\hline \multirow[t]{6}{*}{ Antibiotic and toxin controls } & Penicillin-G & NT & - & NT & - & - & - \\
\hline & Erythromycin & NT & - & NT & - & - & - \\
\hline & Tetracycline & NT & 1.25 & NT & 1.25 & - & - \\
\hline & Chloramphenicol & NT & 2.5 & NT & 2.5 & - & - \\
\hline & Potassium dichromate & NT & NT & NT & NT & 58 & NT \\
\hline & Quinine & NT & NT & NT & NT & NT & 87 \\
\hline
\end{tabular}

$\mathrm{M}=$ methanol extract; $\mathrm{W}$ = water extract; $\mathrm{E}$ = ethyl acetate extract; $\mathrm{DD}=$ disc diffusion; $\mathrm{LD}=$ liquid dilution; $\mathrm{NT}$ = not tested; - indicates no inhibition or not toxic at any dose tested.

Table 3: Qualitative HPLC-MS QTOF and GC-MS headspace analysis of the most promising Terminalia spp. methanolic leaf extracts extract, elucidation of empirical formulas and putative identification (where possible) of the compounds. 


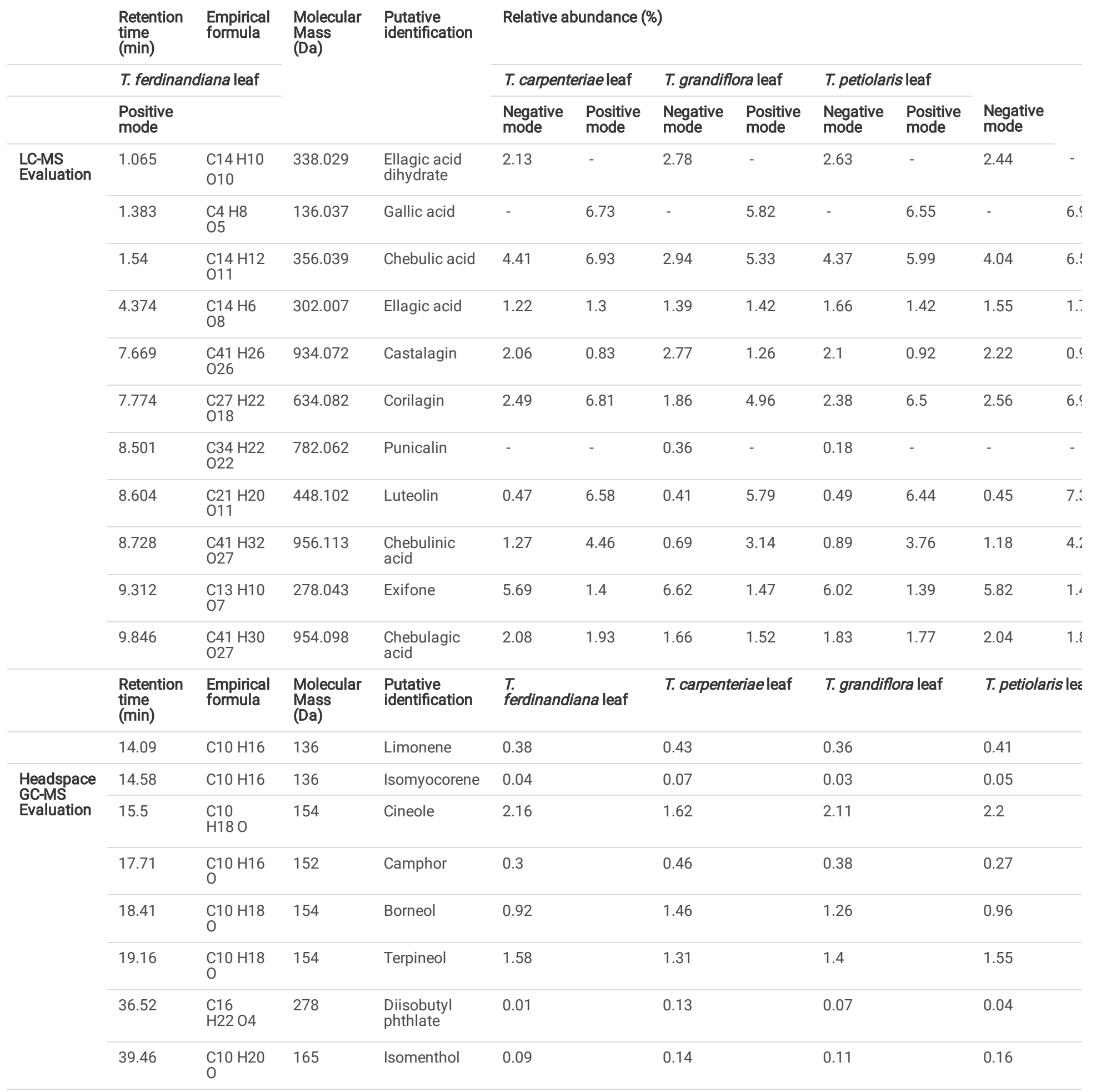

The relative abundance expressed in this table is a measure of the area under the peak expressed as a \% of the total area under all chromatographic peaks.

\section{Figures}



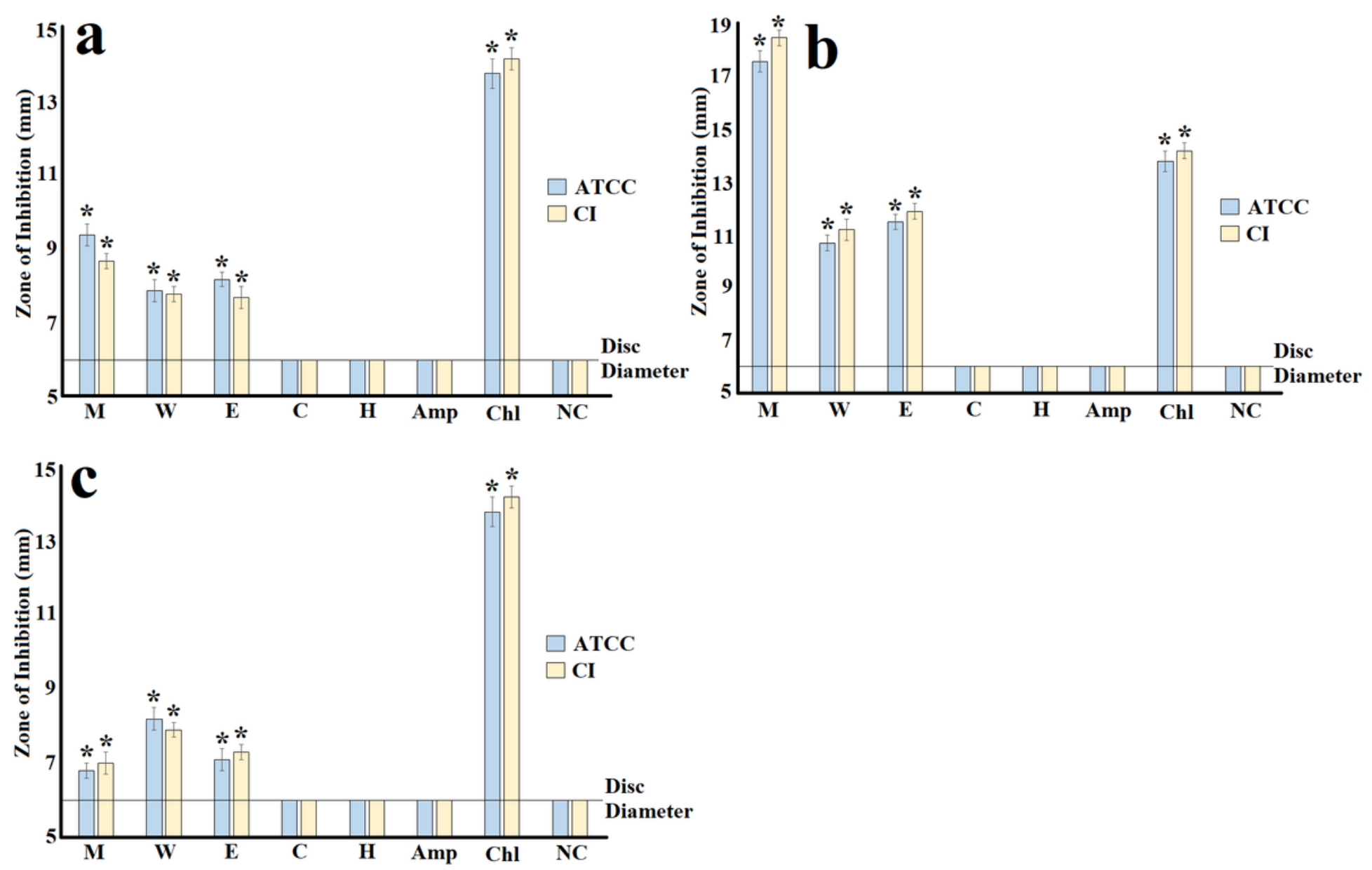

Figure 1

Growth inhibitory activity of the Terminalia ferdinandiana (a) fruit, (b) leaf and (c) seed extracts measured as zones of inhibition (mm) against reference (ATCC31488) and clinical isolate strains of K. pneumoniae. Inhibition zones are represented as the means of three independent experiments, each with internal triplicates $(n=9) \pm$ SEM. $M=$ methanolic extract; $W=$ aqueous extract; $E=$ ethyl acetate extract; $C=$ chloroform extract; $H=$ hexane extract; $A m p=$ ampicillin control $(10 \mu \mathrm{g}) ; \mathrm{Chl}=$ chloroform control $(10 \mu \mathrm{g})$; ATCC and $\mathrm{Cl}$ refer to the reference and clinical bacterial strains respectively; $\mathrm{NC}=$ negative control. 


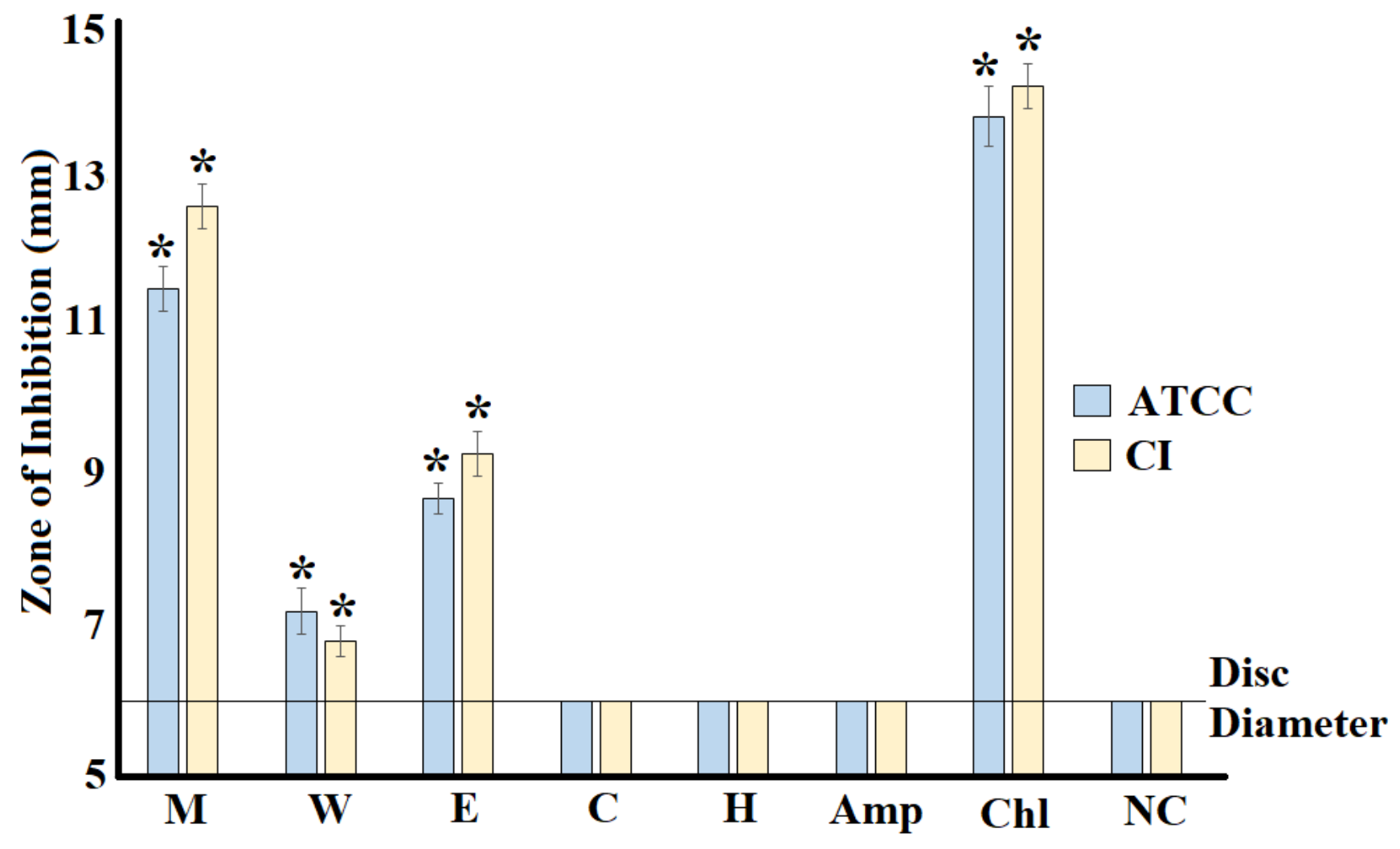

Figure 2

Growth inhibitory activity of the Terminalia carpenteriae leaf extract measured as zones of inhibition (mm) against reference (ATCC31488) and clinical isolate strains of K. pneumoniae. Inhibition zones are represented as the means of three independent experiments, each with internal triplicates $(n=9) \pm S E M$. $M=$ methanolic extract; $\mathrm{W}=$ aqueous extract; $\mathrm{E}=$ ethyl acetate extract; $\mathrm{C}=$ chloroform extract; $\mathrm{H}=$ hexane extract; $\mathrm{Amp}=$ ampicillin control $(10 \mu \mathrm{g}) ; \mathrm{Chl}=$ chloroform control $(10 \mu \mathrm{g})$; $\mathrm{ATCC}$ and $\mathrm{Cl}$ refer to the reference and clinical bacterial strains respectively; $\mathrm{NC}=$ negative control. 

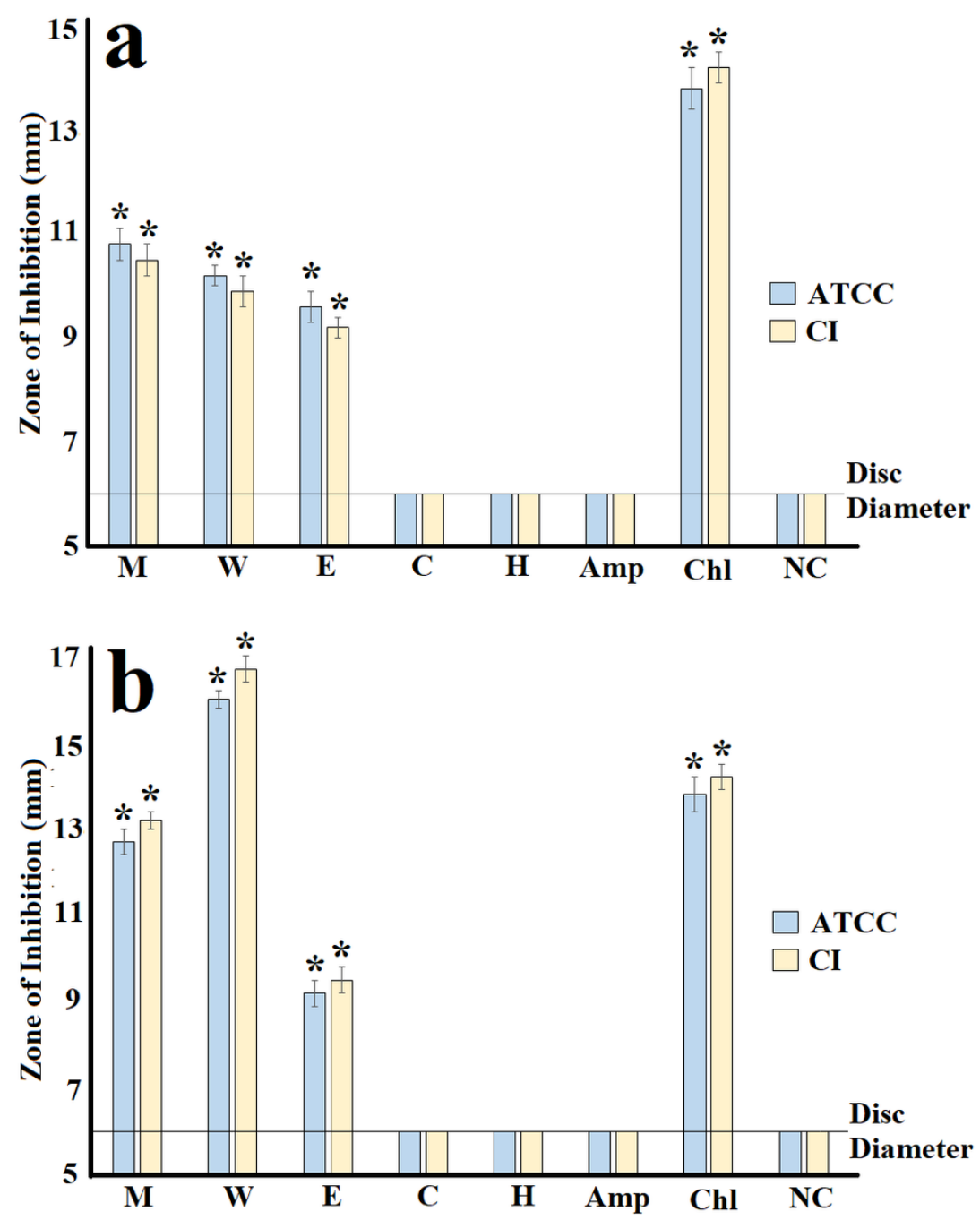

Figure 3

Growth inhibitory activity of the Terminalia grandiflora (a) fruit and (b) leaf extracts measured as zones of inhibition (mm) against reference (ATCC31488) and clinical isolate strains of K. pneumoniae. Inhibition zones are represented as the means of three independent experiments, each with internal triplicates ( $\mathrm{n}=9) \pm$ SEM. $M=$ methanolic extract; $\mathrm{W}=$ aqueous extract; $\mathrm{E}=$ ethyl acetate extract; $\mathrm{C}=$ chloroform extract; $\mathrm{H}=$ hexane extract; $\mathrm{Amp}=$ ampicillin control (10 $\mu \mathrm{g}) ; \mathrm{Chl}=$ chloroform control $(10 \mu \mathrm{g})$; ATCC and $\mathrm{Cl}$ refer to the reference and clinical bacterial strains respectively; $\mathrm{NC}=$ negative control. 

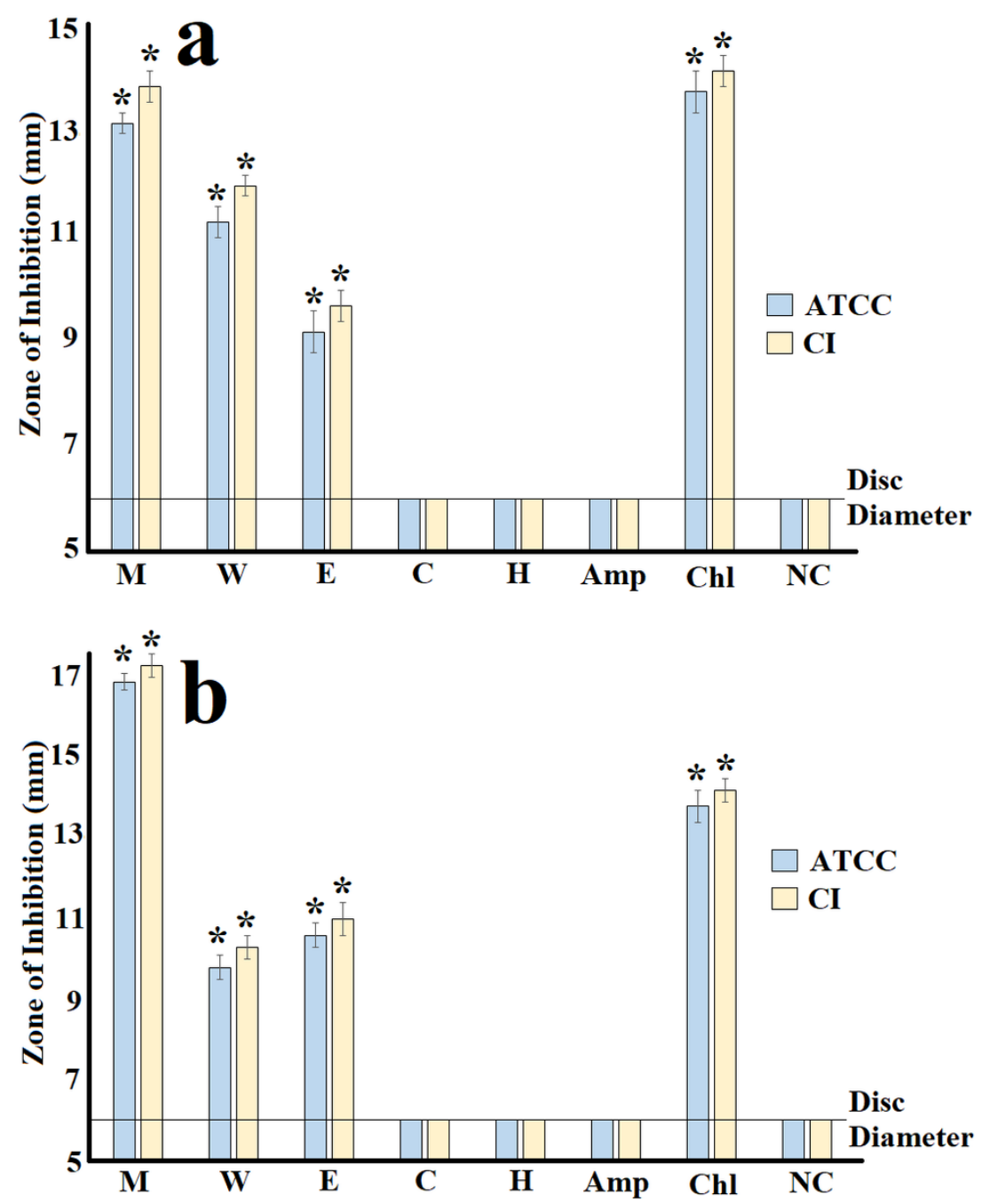

Figure 4

Growth inhibitory activity of the Terminalia petiolaris (a) fruit and (b) leaf extracts measured as zones of inhibition (mm) against reference (ATCC31488) and clinical isolate strains of K. pneumoniae. Inhibition zones are represented as the means of three independent experiments, each with internal triplicates ( $\mathrm{n}=9) \pm$ SEM. $\mathrm{M}=$ methanolic extract; $\mathrm{W}=$ aqueous extract; $\mathrm{E}=$ ethyl acetate extract; $\mathrm{C}=$ chloroform extract; $\mathrm{H}=$ hexane extract; $\mathrm{Amp}=$ ampicillin control (10 $\mu \mathrm{g}) ; \mathrm{Ch}$ = chloroform control $(10 \mu \mathrm{g})$; ATCC and $\mathrm{Cl}$ refer to the reference and clinical bacterial strains respectively; $\mathrm{NC}=$ negative control. 

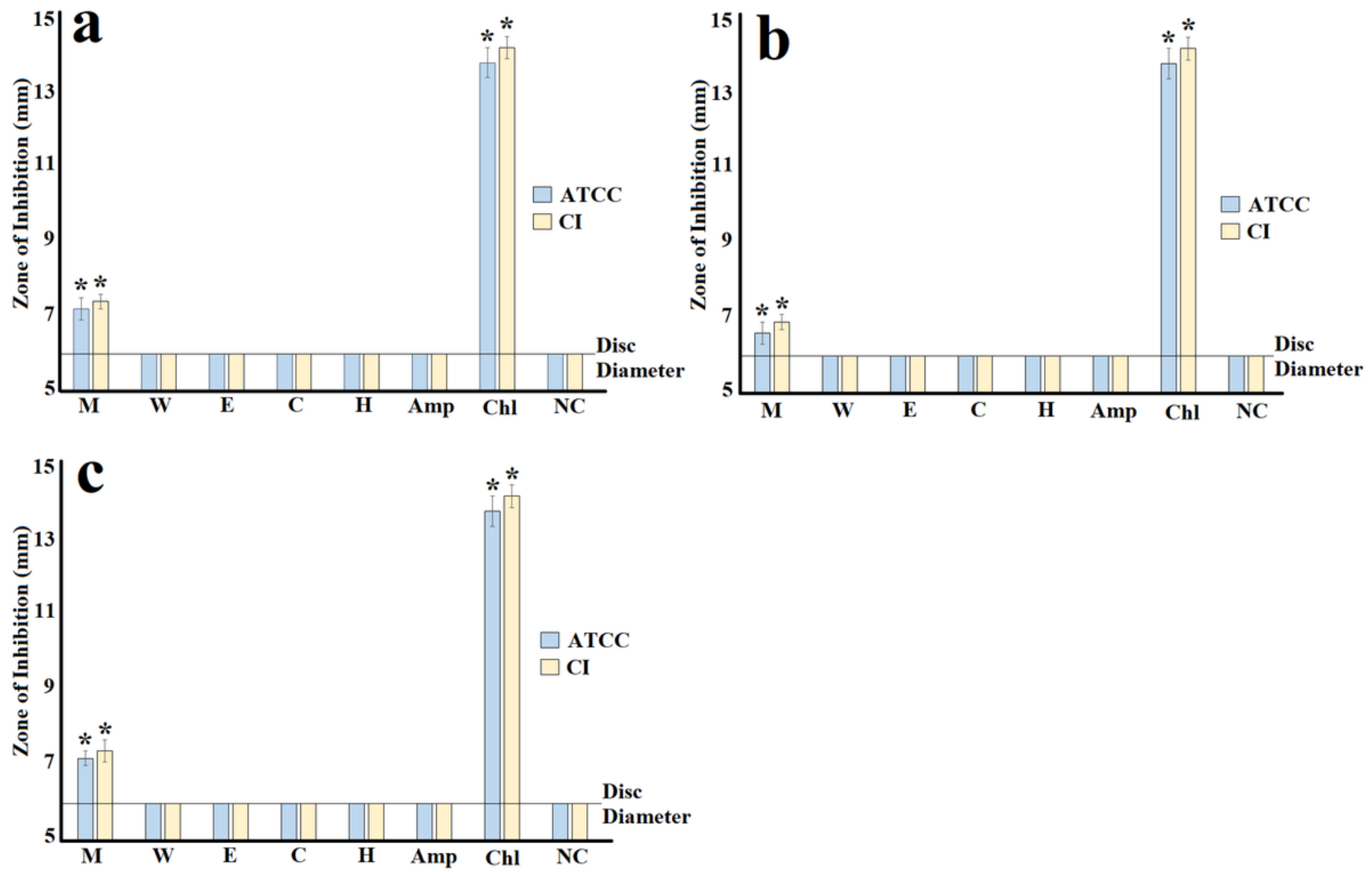

Figure 5

Growth inhibitory activity of the Terminalia catappa (a) bark, (b) fruit and (c) leaf extracts measured as zones of inhibition (mm) against reference (ATCC31488) and clinical isolate strains of K. pneumoniae. Inhibition zones are represented as the means of three independent experiments, each with internal triplicates $(n=9) \pm$ SEM. $M=$ methanolic extract; $W=$ aqueous extract; $E$ = ethyl acetate extract; $\mathrm{C}=$ chloroform extract; $\mathrm{H}=$ hexane extract; $\mathrm{Amp}=$ ampicillin control $(10 \mu \mathrm{g}) ; \mathrm{Chl}=$ chloroform control $(10 \mu \mathrm{g})$; ATCC and $\mathrm{Cl}$ refer to the reference and clinical bacterial strains respectively; $\mathrm{NC}=$ negative control.

\section{Supplementary Files}

This is a list of supplementary files associated with this preprint. Click to download.

- Table1.docx 